\title{
SCIDiC
}

International Journal of Dentistry and Oral Science (IJDOS)

ISSN: 2377-8075

\section{The Corona Chronicle - From History To Hitherto - A Perspective}

Research Article

Aravind Kumar Subramanian ${ }^{1 *}$, Nivethigaa B ${ }^{2}$, Vivek Narayanan ${ }^{3}$

${ }^{1}$ Professor and Head Department of Orthodontics and Dentofacial orthopedics,Saveetha Dental College, Saveetha Institute of Medical and Technical Sciences, SIMATS,Chennai, India.

${ }^{2}$ Senior Lecturer, Department of Orthodontics and Dentofacialorthopedics,Saveetha Dental College, Saveetha Institute of Medical and Technical Sciences, SIMATS, Chennai, India.

${ }^{3}$ Senior Lecturer, Department of Oral Medicine and Radiology,Saveetha Dental College, Saveetha Institute of Medical and Technical Sciences,SIMATS, Chennai, India.

\section{Abstract}

COVID-19 has a global outbreak since its discovery in Hubei province, China in December 2019. Following this it had been declared as a "Public Health Emergency of International Concern" on 30th January 2020 by the World health Organization (WHO). Corona viridae, the causative species that is not new to the society, yet poses serious threat to life in under developed to well-developed nations. First occurrence of such enveloped, non-segmented, single-stranded RNA viruses with club shaped projections was cited back in 1930's attributing to cause respiratory infection in domesticated chickens. The human corona viral illness was eminently noted around 1960's wherein healthy human volunteers were relied on to detect the virus involved in the causation of common cold, an infection of the upper respiratory tract. Tracing back the etiology of such sickness, emergence from the bats and transmission and adaptation in other mammals have occurred which had further led to extensive layout of the malady. It was after the year 2003 when the human population was heavily affected, these infections were thrown to limelight indicating the fierce nature of this mutated subspecies. Since then so many disease variants have been identified including Severe Acute Respiratory Syndrome (SARS-CoV), the Middle Eastern Respiratory syndrome (MERS$\mathrm{CoV}$ ), Bat corona virus (RaTG13) and the COVID 19 (The novel coronavirus- initially named to be SARS-CoV-2). Not just the upper respiratory tract anymore, this disease had turned out to be more aggressive affecting the entire system causing a complete shutdown. Knowing the various possible modes of spread of this contagious disease, dental fraternity poses most risk due to transmission through contact, especially the aerosols. But the atypical nature of this virus, with increasing number of mutated subspecies no possibility exists in using a routine antiviral drug to completely eradicate this infection. Diagnostics can play an important role in the containment of COVID-19, enabling the rapid implementation of control measures that limit the spread through case identification, isolation, and contact tracing.

This chapter focuses on the epidemiological report, the pathogenesis involved and the sequalae of occurrence of coronal viral disease in humans with an update on the recent one, a brief overview of various diagnostic tools utilized including Molecular assays,Nucleic acid testing (RT-PCR),CT Scans and a dental outlook on how the spread occurs along with methods by which dental setup could be immunized to intercept any further viral ailment.

Keywords: COVID; Corona Virus; Pandemic; Pathogenesis; SARS CoV2; Respiratory Tract Infection; Dental Implications.

\section{Background}

The word pandemics isn't new to this society. Since the time mankind evolved several new infections have been identified every now and then. It ranged in severity from mild flu to several deadly infectious diseases [1-6]. Plague, a bacterial disease first noted years ago around 1 st century $\mathrm{AD}$ in several countries around the world [7]. The cholera had been deadlier in almost all the continents [8]. One major factor eminent in all these was that episodes of recurrence happened decades after the original outbreak.

*Corresponding Author:

Aravind Kumar Subramanian,

Professor and Head Department of Orthodontics and Dentofacial Orthopedics, Saveetha Dental College, Saveetha Institute of Medical and Technical Sciences, SIMATS, Chennai, India.

Tel: 9841299939

E-mail: aravindkumar@saveetha.com

Received: December 27, 2020

Accepted: January 11, 2021

Published: January 15, 2021

Citation: Aravind Kumar Subramanian, Nivethigaa B, Vivek Naravanan. The Corona Chronicle - From History To Hitherto - A Perspective. Int J Dentistry Oral Sci. 2021;8(1):13791385. doi: http://dx.doi.org/10.19070/2377-8075-21000273

Copyright: Aravind Kumar Subramanian ${ }^{\circ} 2021$. This is an open-access article distributed under the terms of the Creative Commons Attribution License, which permits unrestricted use, distribution and reproduction in any medium, provided the original author and source are credited. 
Ensuing incidences were due to some mutated subspecies which proved to be even lethal than the original disease itself $[9,10]$. Similar outburst happened in 2003 when the corona viral infection was first noted in human beings [11]. Flare-up of the mutated form of this viral infection had occurred in many nations far off from the original epicenter of the disease [12-14]. China reported an endemic viral infection among the residents of Wuhan, Hubei province, China around mid-December 2019. Although initially this disease was believed to contained within that particular geographical region it had rapidly spread to the other countries. Analyzing the scenario then, World health Organization announced it as a "Public Health Emergency of International Concern" on 30th January 2020. From then so many countries around the world has been alarmed of its fast spread and detrimental nature. Even three months after this, till date the spread of viral infection couldn't yet be controlled.

Occurrence of Corona viridae (the causative organism), an enveloped, non-segmented, single-stranded RNA virus with club shaped projections was cited back in 1930's attributing to cause respiratory infection (zoonotic) in domesticated chickens [15, 16]. The human corona viral illness was eminently noted around 1960's wherein healthy human volunteers were relied on to detect the virus involved in the causation of common cold, an infection of the upper respiratory tract [17]. Tracing back the etiology of such sickness, emergence from the bats and transmission and adaptation in other mammals have occurred which had further led to extensive layout of the malady [18].

\section{Epidemiology of the Outbreak}

Initially these cases presented with symptoms similar to pneumonia which included fever, difficulty in breathing, dry cough with a ground glass appearance of alveoli in both the lungs. The shanghai public health center along with the central hospital of Wuhan, released the Novel corona virus genomic sequence, which was believed to have caused the outbreak in the Hubei province [19].

On 31st of December, the Wuhan municipal health commission had put forward a formal statement indicating the spread of viral infection of unknown etiology in the Hubei province. It remained unclear regarding the antecedent involved in causation of this disease. The origin of this disease was then believed to be the seafood market in that location [20]. When fever and pneumonia was witnessed in cluster of cases at Wuhan Jinyintan Hospital, Broncho-alveolar lavage was performed, samples were collected and tested, it revealed SARS like corona virus strain, Bat CoV, RaTg 13 which showed almost 96\% identity. Following isolation from cell lines, the appearance of crown like particles were confirmed which was then identified as SARS CoV2. Also, postmortem samples confirmed widespread alveolar damage in both the lungs with cellular exudates of fibro-myxoid type. Evidences were in favor of Acute respiratory distress syndrome(ARDS) [21].

The number rose on 2nd of January when 41 patients who presented with similar symptoms were confirmed positive with nCOV infection. Ratio between male and female showed greater incidence of the disease in the male population (male being $73 \%$ among the overall infected people). Also, the mean age was then found to be 49 years. The prevalence of other systemic diseases like diabetes, hypertension and cardiovascular disease in exposed individuals posed a risk for developing extreme life-threatening symptoms among exposed individuals.

At the start, seeing the nosocomial spread of the disease, this disease was thought to have a less aggressive spread. Since 16th of January 2020 there was a 10 -fold increase in the total number of reported cases and cumulative score for the number of people rose to 440 on 22nd January 2020 [22].

\section{Modes of Transmission}

Not just for corona virus, any contagious viral infection can have direct or indirect spread. Direct spread of the virus includes transmission through actual contact with infected person and various secretions from them such as cough, sneeze or respiratory droplet. Indirect spread occurs through contact transmission with particles inhalation of aerosol contaminated with the virus, or any other surfaces where the infected person had been in contact with. Few of these are till now confirmed with human corona virus transmission. Respiratory spread of the virus has occurred with contact either directly with droplets of different sizes and through saliva directly or indirectly. Few cases have also been identified to be caused by asymptomatic carriers, like on in the Germany [23]. Medical procedures result in the synthesis of aerosol particles which become carrier of infection to the health care workers. The alpha and beta forms of the virus are said to use respiratory system as a main mode for transmission wherein the gamma and delta forms of the virus were transmitted through the fecal mode. Few studies suggest that the fecal route of transmission is possible for the mutated corona species and hence it can pose a problem to sanitation workers which can build up the chain of spread of this viral infection. In a study on the first few infected cases in United States of America it results from r RTPCR test for the human stool have confirmed the presence of virus in the stool sample on the $7^{\text {th }}$ day in the active phase of coronal viral disease [24].

\section{Clinical Features}

The initial symptoms included fever, cough, muscle pain and tiredness; infrequent symptoms that occurred in very few cases were cough with sputum, headache, hemoptysis, diarrhea, new loss of taste sensation, hemoptysis, dyspnea and lymphopenia [26]. Almost all the patients experienced difficulty in breathing and presented with a classical ground class appearance on CT with pneumonia like symptoms [27].

There was a mean 10 day delay between exposure and presentation of clinical signs and symptoms, with an average of 5-6 day incubation period and 4-5 delay in hospitalization of infected individuals. [28]. From the period of onset, death occurred in about 6 to 41 days based on the severity of the condition. More deceased were found to be the elderly population above the age group of 70 years and also those who had other systemic ailments.

Emergency medical care should be sought if the patient experiences difficulty in breathing, diffuse and prolonged pain in the chest region, delirium, bluish discoloration of the lips and the face [29]. IL2, IL7, GCSF, IP10, MCP1, MIP1A and TNF alpha were found higher in patients who were under ventilator assisted breathing. Vascular lesions and skin lesion were reported in a few 
cases [30].

\section{Complications and Clinical Outcomes}

In COVID 19 positive cases apart from the acute respiratory distress syndrome patients also developed other complications including arrhythmia, shock, acute cardiac injury, kidney injury, liver dysfunction and few other secondary infections [31-36]. Stage III of the disease progression usually results in such multi organ failure especially in elderly people and neonates whose inbuilt innate immune system is week to respond to the viral attack [27].

\section{Criteria to Assess Severity of COVID - 19}

Mild cases: Cases that has remained in stage 1 or 2 without further progression. Only mild clinical symptoms persist with no cough or respiratory findings. Imaging doesn't provide any detail in this stage.

Moderate cases: Fever, appearance of respiratory symptoms and ground glass appearance in the chest radiograph [37].

Severe cases: difficulty in breathing, partial pressure of oxygen drops to less than $300 \mathrm{mmHg}$ and rapid timely changes appearing in the successive radiographs. Based on these findings need for vigorous treatment is decided [38].

Critical cases: Inability to respire without the help of a ventilator, respiratory and cardiogenic chock with a multi organ failure. Need for extensive monitoring is required with ventilator assisted breathing [39].

\section{Radiographic Diagnosis}

Radiological assessments are vital in the discovery and the management of COVID-19[40]. Ground glass opacity was seen in the chest radiographs in advanced stage but during the initial stage of the disease this feature is not evident. Hence chest radiography is not recommended as the confirmatory imaging modality for initial diagnosis of COVID-19. Significant number of clinicians proposed CT scan ought to be one vital assistant analytic strategy since it is increasingly sensitive in determining the severity [41, 42].

The chest CT findings include ground glass opacities (GGO), peripheral subpleural distribution, patchy consolidations and crazy paving pattern (GGO with superimposed inter and intra-globular septal thickening) [43]. GGO is a hazy increase in attenuation that appears in a variety of interstitial and alveolar processes with pres-

Figure 1. Cumulative case report (as on 19-11-2020).

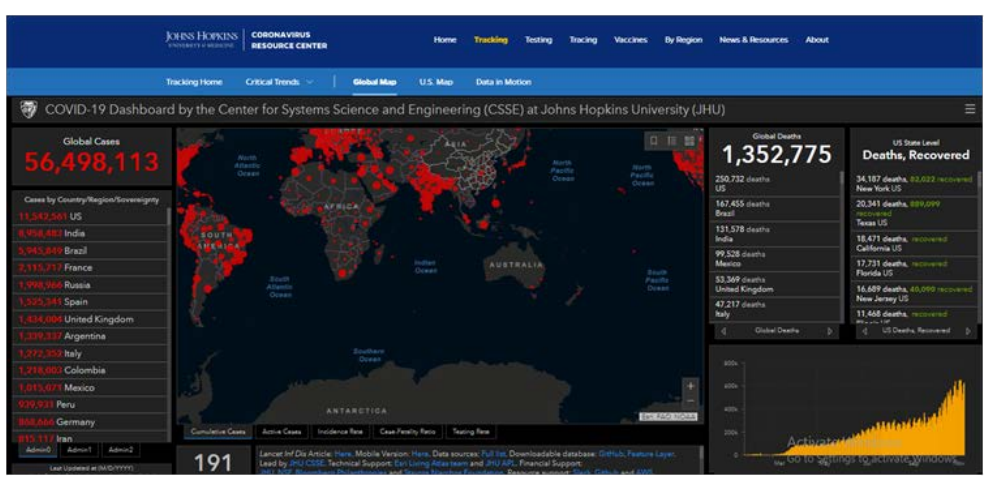

Figure 2. Patient Screening Protocol during a Pandemic [53].

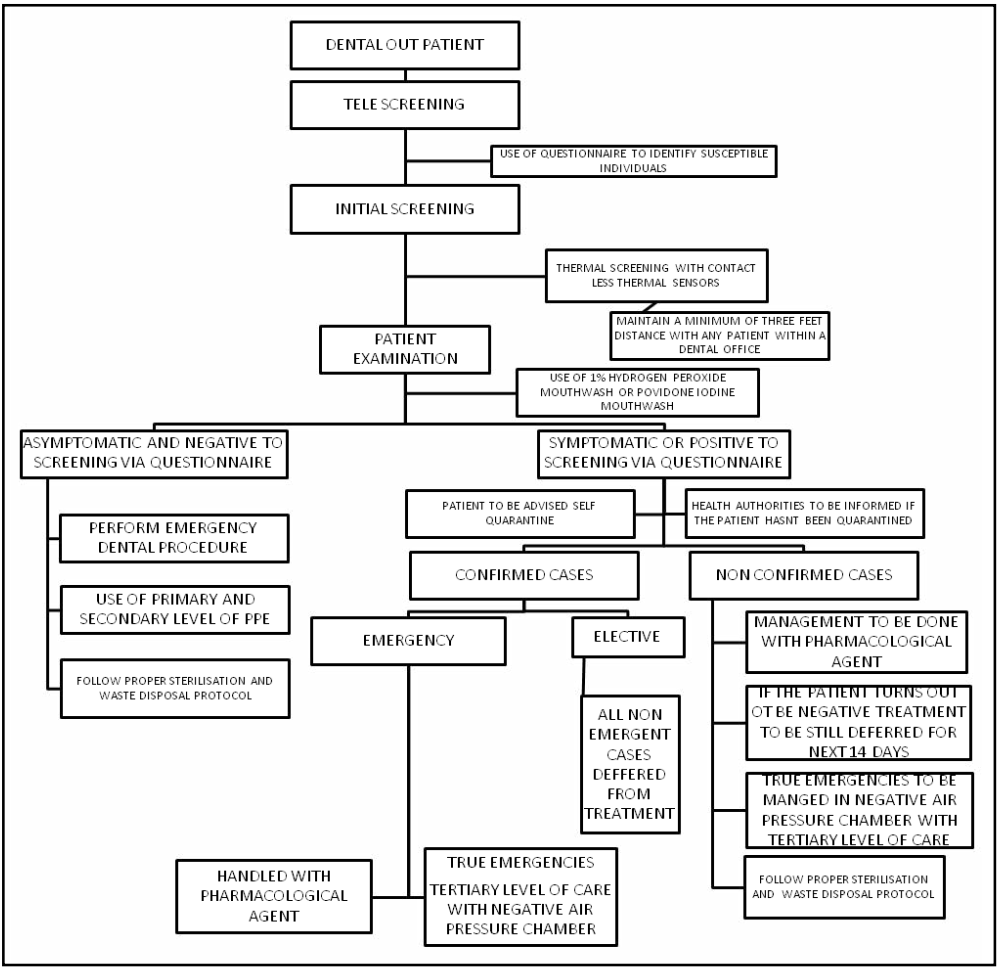


Figure 3. Standard protocol regarding the use of personal protective equipment.
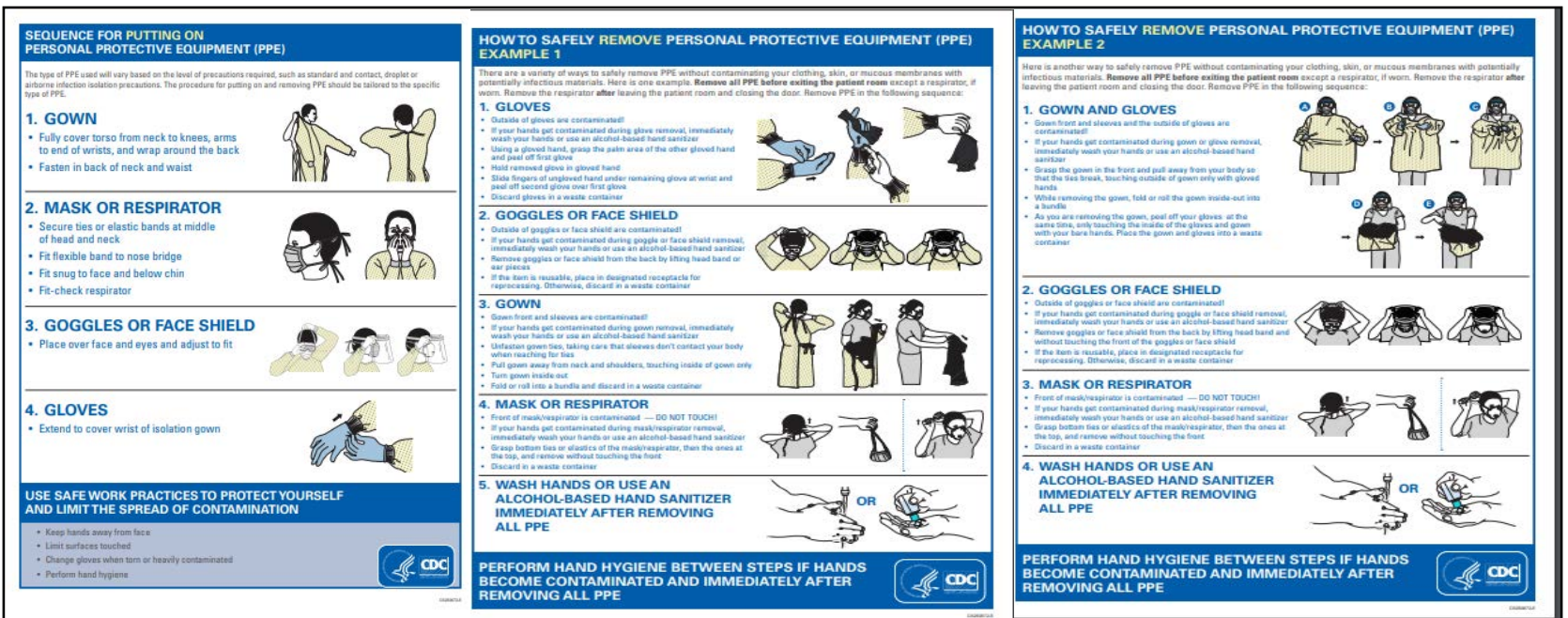

ervation of bronchial and vascular margins [44]. High resolution CT (HRCT) for the chest is basic for early finding and assessment of sickness seriousness of patients with SARS-CoV-2 45. According to the fifth trial version chest CT discoveries of viral pneumonia are viewed as the clinical finding of COVID - 19 disease. In the initial stages of the pneumonia pure GGO appearance can be present. Pleural effusion, lung cavitation, lymphadenopathy and calcification are infrequently reported [44]. Diseases which appear similar to pneumonia must be distinguished from COVID-19. The other causes of pneumonia include streptococcus pneumonia, chlamydia pneumonia and older coronavirus infections. The COVID-19 also mimics certain other diseases such as common cold, influenza, SARS and MERS. Thin slice CT is recommended which aids not only in diagnosis but also to determine the extent of the disease. Even though CT findings are non-specific they significantly aid in the diagnosis of COVID-19.

\section{Laboratory Diagnosis}

The virus spread through the respiratory mucosa and contaminate different cells, initiate a cytokine storm in the body, produce a progression of resistant reactions, and cause changes in peripheral WBCs and immune cells, for example, lymphocytes [46, 47]. Patients might show normal WBCs or leukopenia, lymphopenia, or thrombocytopenia, with prolonged activated thromboplastin time and high C-reactive protein [44]. RT-PCR stands for reverse transcription polymerase chain reaction. At present, RT-PCR test remains the reference standard to make a complete conclusion of COVID-19 contamination [48]. The test involves reverse transcription of SARS-CoV-2 RNA into complementary DNA (cDNA) strands, followed by amplification of specific regions of the cDNA [49]. The measure can be structured as a two-target framework, where one primer recognizes various coronaviruses counting SARS-CoV-2 and a subsequent primer set just recognizes SARS-CoV-2.

RT - PCR can be a single step or a dual step process. In the former the reverse transcription and PCR amplification are grouped into a single reaction. In the latter the reaction is done separately. The dual step process is more sensitive but also more time consuming. The samples are obtained from the upper respiratory tract and is the broadly recommended sample. Those who exhibit symptoms of a productive cough lower respiratory samples are obtained.
The upper respiratory samples include nasopharyngeal swabs, oropharyngeal swabs, nasopharyngeal washes, and nasal aspirates. Lower respiratory tract samples include sputum, and tracheal aspirates. In the initial 14 days after beginning, SARS-CoV-2 could most dependably be distinguished in sputum followed by nasal swabs. There are three issues that have emerged with RT-PCR. To start with, the accessibility of PCR reagent packs has not kept up with request. Second, community clinics outside of urban communities come up short on the PCR foundation to oblige high test throughput. Finally, RT-PCR depends on the nearness of perceptible SARS-CoV-2 in the sample gathered. COVID-19 is presently determined by RT-PCR and has been screened for with CT scans, however both the procedure has its own downsides $[50,51]$.

\section{Differential Diagnosis}
a) Common cold
b) Influenza
c) SARS
d) MERS
e) Chlamydia pneumoniae
f) Human meta-pneumonia virus
g) Human rhinovirus
h) Adenovirus
i) Primary viral or bacterial pneumonia

\section{Dental Office A Potential Hotspot for Spread of The Virus}

From the available resources on mode of transmission of the viral infection it is very clear that the dental office can be a hotspot since transmission can occurs through direct contact with an infected patient to the dental health care workers or vice versa. The reason being the long incubation period before the active phase of the infection which has extended to 14 days after the initial time of contamination with the pathogen on the host, hence increasing the difficulty for the doctor to readily isolate these cases. Studies have shown the spread through saliva, which here proves to be the most common mode of transmission. This can happen when the cough or sneeze from the infected person had been contacted with. The corona virus has been shown to have a higher 
affinity towards the Angiotensin converting enzyme (ACE) receptors. Based on the coding studies done, it had been presumed than the ACE receptors predominate in number in the salivary gland than in the lungs. Confirmatory studies on this fact had fetched eye opening particulars that salivary glands(major and minor) are reservoirs for the corona virus especially among the asymptomatic carriers [52].

\section{Air and Contact Borne Infection}

Literatures have given a clear note on spread of the corona viral disease. It becomes inevitable to avoid all these while treating a patient. The concept of using a face mask and social distancing isn't applicable in a dental setup. Spread can occur through the droplets propelled when the patient sneezes or coughs without mask while examining or while performing treatment and also the aerosol that is generated while doing a dental procedure. Not just the oral secretions but also exposure to conjunctival, nasal secretions and also the blood contamination from the infected patients can happen with the aerosols that are generated through the use of micromotor and aerator handpiece. These particulate materials are formed in huge amount that the dental office becomes completely infected after procedures are done. Apart from this airborne direct spread, indirect spread can also occur in case of improper disinfection of the contaminated instruments. Understanding the different modes of spread of the disease, the dental setup is a highly susceptible place for acquiring and transmission of diseases, especially the aerosol borne particulate matter. The viral particles remain suspended over a longer period time of about 2 hours to even 9 days and a relative humidity of $50 \%$ it is more virulent than at $30 \%$. Hence spread of such infection can be avoided by maintaining clean and dry environment.

\section{Decision on need for Dental Treatment}

Dentist should be properly trained to manage such pandemic conditions. They must have the ability in diagnosing a case for Novel corona virus and isolating them. If in case they happen to identify any new case, then the infection control department has to be alerted regarding this issue and the proper preventive and interceptive measures to be taken care of to prevent spread outside the dental office and within the health care workers. Dental treatment can be emergency care or elective care. Any patient in the active phase of the disease is completely unfit to undergo dental treatment. Almost all elective procedures can be postponed in patients during this period. But a dental emergency can arise anytime. Under such circumstances to act with caution is highly necessary to protect the dentist, dental assistant and the other patients who visit the dental fraternity.

\section{Patient Examination and Isolation}

Teleconferencing with the patient should be followed as the first step in any outpatient set up. Patient should be made aware about the seriousness of the current situation, asked for subjective signs of any active disease and also any recent history of travel history or possibility in being within the epidemiological link. For this preparing a questionnaire and following it for all patients will help us avoid negligence. This questionnaire should be filled in teleconferencing or when the patient enters the dental clinic before any examination is even started.
This should include the following criteria to be asked for:

1. Any history of recent illness including sore throat, fever, cough or rhinitis or other respiratory problems.

2. Any history of recent travel abroad

3. Any history of recent travel within state.

4. Any history of contact with people infected with the disease.

5. Any history of infection in the neighborhood.

6. Any history of active infection within family members.

7. Any history of recent participation in groups or gatherings.

If any these questions turn out to be positive patient is advised to check for any active viral infection or remain in self-quarantine for the next 14 days. Patients' further visit to the dental office is deferred until further notice from the health authorities on the status of the patient.

For walk in patients, the patient is systematically analyzed at the moment patient enters into the dental office. Patient should be made sure that their temperature is within normal range of $<100$ F. For this contactless heat sensors should be used.

1. If any of these turns out to be positive like a patient experiencing any symptoms or those having a history of travel to COVID affected regions,

a. In case of life-threatening emergent situation patient can be allowed to come to the hospital set up but has to be treated with special PPE precautions and separate waste protocol and negative pressure chamber.

b. If otherwise, for situations that can be brought under control with pharmacological agents, appropriate prescription can be given such that the patient returns to the dental office after he is tested negative for COVID (i.e. after two weeks preferably) and immediate dental treatment is avoided.

c. Elective dental procedures can be completely avoided and the patient should be well educated regarding the lesser need of treatment at that point in time.

2. For those patients without any history of travel, possible epidemiological link or symptoms, dental treatment can be performed. But treatment involving the aerosol production can be deferred till the outbreak is brought under control, for the sake of remaining within the safety margin.

3. If the use of aerosol generating procedure becomes a part of necessary emergency care, then all the precautionary measures should be properly taken care off including the use of high-pressure suction for evacuation and proper isolation protocol involving rubber dams.

\section{Immunization Protocol For Routine Dental Prac-} tice

\section{i. Personal protective equipment:}

Earlier we did not have any proper guidelines for protective care for COVID 19. Recently it had been put forth by dental council existing in various countries. Few cases of COVID 19 have also been identified among dentists who treated asymptomatic 
infected patients, insisting the need to follow such council made protocol and guideline. Considerations have been given in terms of hand hygiene, use of personal protective equipment i.e., gown, eye protection goggles, and Facemask (N95 or higher-level respirator), their disposal and reuse strategies should be properly followed according to the CDC protocol and also the manufacturers instruction [54]. Personal protective equipment to be worn while treating all patients preferably a sterilized one for each patient. Based on the spread of infection and increased need for control the wear of personal protective equipment has been subdivided into three categories $[55,56]$.

In general cases which do not have any history, and the questionnaire is negative, Primary protection, use of facemask, gloves with a regular use sterilizable protective coat for the dental assistant. Advanced secondary protection can be attained for the dentist by the wear of facemask, surgical gloves, headcap, protective face shield, regular use protective coat along disposable isolation clothes on the outside. Tertiary level of care is required if the patient is in the active phase of the disease or under self-quarantine or if the questionnaire turns out to be positive. These patients are not advised to reach for any dental setup for elective treatments but in case of emergency extreme levels of protection should be followed by the dentist and the dental assistants and the number of people in the working area should be kept as minimum as possible $[57,58]$.

ii. Hand Hygiene: The route of transmission had made it compulsory to follow extensive handwashing techniques. It is very essential to not acquire or transmit the disease and also not be a potential carrier of transmission to others. Health advisories have advised following these protocols every time someone visits a new place or been in contact with another person. This same applies to a dental facility as well. In the clinic handwashing is followed by the patient when they enter in, before any procedure or after the procedure is over. And the dentist is advised to follow hand hygiene before examining the patient, before starting any procedure, after the procedure is completed and when contacting with any dental unsterilized instruments or surfaces or if they come in contact with blood saliva or other droplet anytime during the entire procedure. Care to be taken in avoiding contact with eye, nose and mouth anytime between the procedures, before an alcohol rub sanitizer is used.

iii. Mouth rinsing: Procedural mouth rinse with $1 \%$ hydrogen peroxide or povidone iodine is suggested to reduce the oral viral load and making it a better environment to carry out emergency procedures.

iv. Disposable instruments: Instead of the regular use stainless steel mouth mirror and probe for examination and isolation, it is better to use a disposable mouth mirror, probe, disposable needles and single use materials on the patients.

v. Rubber dam isolation: With the aim of avoiding blood and saliva contamination with the aerosol, use of proper method of isolation such as a rubber dam kit can be considered. Aerosol that is generated can be evacuated using a high-volume evacuator that is used in case of surgical procedures. Those which leads to aerosol production should be avoided. Use of caries dissolving agents, atraumatic restorative treatment without handpiece and also hand scalers to be preferred over modernized aerosol gen- erating procedures.

vi. Radiographs: Use of extraoral radiographs to be preferred over intraoral radiographs and also when intraoral radiographs are indicated, use of proper double layered barrier technique to be followed.

vii. Airborne infection isolation chambers: All suspected and confirmed cases, to be treated in negative pressure rooms or air borne infection isolation chambers. But this cannot be provided in a regular dental setup. Hence it would be more reasonable to treat these dental emergencies in a medical setup if the patient is either tested positive for COVID 19 or quarantined as a susceptible individual. The dental emergencies in such conditions include only the maxillofacial trauma or space infections like cellulitis, dentoalveolar abscess etc., which would turn into a life-threatening emergency if left untreated.

viii. Disinfection of dental office: Every time a patient moves out of the dental clinic, it is the responsibility of the dental assistant to clean all the in animate surfaces so that the viral spread through contact with these can be avoided. The dental assistant should be trained adequately regarding the importance of disinfection and sterilization twice as fold as in every day dental practice.

ix. Anti-retraction devices: In few cases even there is a chance of retrograde contamination of the waterline that supply the chair. This can act as potential reservoir to spread infection. Need for anti-retraction devices is vital at this point. Handpieces without anti retraction devices should be prohibited from use. And newer inventions should be made for the other dental water supplies also so that any retrograde infection can thus be prevented.

x. Dental waste management: Categorization of the dental waste need to be done and disposed in sealed container to prevent the spread of infection among the sanitation workers who collect and dispose the waste.

xi. In case of any sickness reported among the dental assistants, they must be strictly advised not to come to the dental office and handle any clinic related materials. If normal flu like symptoms subside in a day or two, then they can return back to work once they are normal. But in case if it still persists, they might be advised to maintain quarantine and seek adequate professional help.

\section{Acknowledgement}

The authors would like to extend their gratitude to Saveetha Dental and Hospital for providing a platform for this research.

\section{References}

[1]. bio061i.pdf. Accessed September 5, 2020. https://www.medigraphic.com/ pdfs/revbio/bio-2006/bio061i.pdf

[2]. Horimoto and Kawaoka - 2005 - INFLUENZA LESSONS FROM PAST PANDEMICS, WARNINGS F.pdf. Accessed September 5, 2020.

[3]. Modelling to contain pandemics | Nature. Accessed September 5, 2020.

[4]. Tumpey TM, Basler CF, Aguilar PV, Zeng H, Solórzano A, Swayne DE, et al. Characterization of the reconstructed 1918 Spanish influenza pandemic virus. Science. 2005 Oct 7;310(5745):77-80.Pubmed PMID: 16210530.

[5]. Hsieh YC, Wu TZ, Liu DP, Shao PL, Chang LY, Lu CY, et al. Influenza pandemics: past, present and future. J FORMOS MED ASSOC. 2006 Jan 


\section{1;105(1):1-6.}

[6]. America's Forgotten Pandemic: The Influenza of 1918 - Alfred W. Crosby Google Books. Accessed September 7, 2020.

[7]. Butler T. Plague history: Yersin's discovery of the causative bacterium in 1894 enabled, in the subsequent century, scientific progress in understanding the disease and the development of treatments and vaccines. Clin Microbiol Infect. 2014 Mar;20(3):202-9.Pubmed PMID: 24438235.

[8]. (PDF) Cholera Pandemics, 1816-1861. ResearchGate. Accessed September 7, 2020 .

[9]. Chatterjee P, Kanungo S, Bhattacharya SK, Dutta S. Mapping cholera outbreaks and antibiotic resistant Vibrio cholerae in India: An assessment of existing data and a scoping review of the literature. Vaccine. 2020 Feb 29;38 Suppl 1:A93-A104.Pubmed PMID: 31883807.

[10]. Jutla A, Khan R, Colwell R. Natural Disasters and Cholera Outbreaks: Current Understanding and Future Outlook. Curr Environ Health Rep. 2017 Mar;4(1):99-107.Pubmed PMID: 28130661.

[11]. Van Der Hoek L, Pyrc K, Jebbink MF, Vermeulen-Oost W, Berkhout RJ, Wolthers KC, et al. Identification of a new human coronavirus. Nat. Med. 2004 Apr;10(4):368-73.

[12]. Woo PC, Lau SK, Huang Y, Yuen KY. Coronavirus diversity, phylogeny and interspecies jumping. Exp Biol Med (Maywood). 2009 Oct;234(10):111727.Pubmed PMID: 19546349.

[13]. Holmes KV. SARS-associated coronavirus. N Engl J Med. 2003 May 15;348(20):1948-51

[14]. Chafekar A, Fielding BC. MERS-CoV: understanding the latest human coronavirus threat. Viruses. 2018 Feb;10(2):93.

[15]. Structure, Function, and Evolution of Coronavirus Spike Proteins | Annual Review of Virology. Accessed September 7, 2020.

[16]. Brian DA, Baric RS. Coronavirus genome structure and replication. In Coronavirus replication and reverse genetics .2005 (pp. 1-30). Springer, Berlin, Heidelberg.

[17]. Cavanagh D. Coronavirus avian infectious bronchitis virus. Vet. Res. 2007 Mar 1;38(2):281-97.

[18]. Lun ZR, Qu LH. Animal-to-human SARS-associated coronavirus transmission?. Emerg Infect Dis. 2004;10(5):959.

[19]. Wu F, Zhao S, Yu B, Chen YM, Wang W, Song ZG, et al. Severe acute respiratory syndrome coronavirus 2 isolate Wuhan-Hu-1, complete genome. Nature. 2020 Mar;579(7798):265-9.

[20]. Wuhan Municipal Health Commission. Accessed April 20, 2020.

[21]. Lu H. Drug treatment options for the 2019-new coronavirus (2019-nCoV). Biosci. Trends. 2020 Feb 29;14(1):69-71.

[22]. 2020-01-22-COVID19-Report-2.pdf. Accessed April 20, 2020.

[23]. Rothe C, Schunk M, Sothmann P, Bretzel G, Froeschl G, Wallrauch C, et al. Transmission of 2019-nCoV infection from an asymptomatic contact in Germany. N Engl J Med. 2020 Mar 5;382(10):970-1.

[24]. Holshue ML, DeBolt C, Lindquist S, Lofy KH, Wiesman J, Bruce H, et al. First case of 2019 novel coronavirus in the United States. N Engl J Med. 2020 Jan 31

[25]. Mason RJ. Pathogenesis of COVID-19 from a cell biology perspective. Eur Respir J. 2020;55(4):2000607.

[26]. Ren LL, Wang YM, Wu ZQ, Xiang ZC, Guo L, Xu T, et al. Identification of a novel coronavirus causing severe pneumonia in human: a descriptive study. Chin Med J (Engl). 2020 May 5;133(9):1015-1024.Pubmed PMID: 32004165.

[27]. Huang C, Wang Y, Li X, Ren L, Zhao J, Hu Y, et al. Clinical features of patients infected with 2019 novel coronavirus in Wuhan, China. The lancet. 2020 Feb 15;395(10223):497-506.

[28]. Report 1 - Estimating the potential total number of novel Coronavirus (2019-nCoV) cases in Wuhan City, China. Imperial College London. Accessed April 20, 2020.

[29]. CDC. Coronavirus Disease 2019 (COVID-19). Centers for Disease Control and Prevention. Published February 11, 2020. Accessed April 21, 2020.

[30]. Bouaziz JD, Duong TA, Jachiet M, Velter C, Lestang P, Cassius C, et al. Vascular skin symptoms in COVID-19: a French observational study. J Eur Acad Dermatol Venereol. 2020 Sep;34(9):e451-e452.Pubmed PMID: 32339344

[31]. Terpos E, Ntanasis-Stathopoulos I, Elalamy I, Kastritis E, Sergentanis TN, Politou M, et al. Hematological findings and complications of COVID-19. Am. J. Hematol. 2020 Apr 13;95(7):834-847.

[32]. Lodigiani C, Iapichino G, Carenzo L, Cecconi M, Ferrazzi P, Sebastian T, et al. Venous and arterial thromboembolic complications in COVID-19 patients admitted to an academic hospital in Milan, Italy. Thromb Res. 2020 Jul;191:9-14.Pubmed PMID: 32353746.

[33]. Klok FA, Kruip MJ, Van der Meer NJ, Arbous MS, Gommers DA, Kant $\mathrm{KM}$, et al. Incidence of thrombotic complications in critically ill ICU patients with COVID-19. Thromb Res. 2020 Apr 10;191:145-147.

[34]. Long B, Brady WJ, Koyfman A, Gottlieb M. Cardiovascular complications in COVID-19. The Am. J. Emerg. Med. 2020 Apr 18;38(7):1504-1507.

[35]. Filatov A, Sharma P, Hindi F, Espinosa PS. Neurological complications of coronavirus disease (COVID-19): encephalopathy. Cureus. $2020 \mathrm{Mar}$;2(3).

[36]. Kochi AN, Tagliari AP, Forleo GB, Fassini GM, Tondo C. Cardiac and arrhythmic complications in patients with COVID-19. J. Cardiovasc. Electrophysiol. 2020 May;31(5):1003-8.

[37]. Gandhi RT, Lynch JB, del Rio C. Mild or moderate COVID-19. N Engl J Med. 2020 Apr 24.

[38]. Hadjadj J, Yatim N, Barnabei L, Corneau A, Boussier J, Smith N, et al. Impaired type I interferon activity and inflammatory responses in severe COVID-19 patients. Science. 2020 Aug 7;369(6504):718-724.Pubmed PMID: 32661059

[39]. Li K, Wu J, Wu F, Guo D, Chen L, Fang Z, et al. The clinical and chest CT features associated with severe and critical COVID-19 pneumonia. Investig. Radiol. 2020.

[40]. Chen SG, Chen JY, Yang YP, Chien CS, Wang ML, Lin LT. Use of radiographic features in COVID-19 diagnosis: Challenges and perspectives. J Chin Med Assoc. 2020 Jul;83(7):644-647.Pubmed PMID: 32349032.

[41]. Hui TC, Khoo HW, Young BE, Mohideen SM, Lee YS, Lim CJ, et al. Clinical utility of chest radiography for severe COVID-19. Quant Imaging Med Surg. 2020 Jul;10(7):1540-1550.

[42]. Choi H, Qi X, Yoon SH, Park SJ, Lee KH, Kim JY, et al. Extension of coronavirus disease 2019 (COVID-19) on chest CT and implications for chest radiograph interpretation. Radiology. Cardiothoracic Imaging. 2020 Apr 1;2(2): e200107.

[43]. Pan F, Ye T, Sun P, Gui S, Liang B, Li L, et al. Time course of lung changes on chest CT during recovery from 2019 novel coronavirus (COVID-19) pneumonia. Radiology. 2020 Feb 13: 200370.

[44]. Zu ZY, Jiang MD, Xu PP, Chen W, Ni QQ, Lu GM, et al. Coronavirus disease 2019 (COVID-19): a perspective from China. Radiology. 2020 Feb 21:200490

[45]. Pan Y, Guan H, Zhou S, Wang Y, Li Q, Zhu T, et al. Initial CT findings and temporal changes in patients with the novel coronavirus pneumonia (2019-nCoV): a study of 63 patients in Wuhan, China. Eur Radiol. 2020 Jun;30(6):3306-3309.Pubmed PMID: 32055945.

[46]. Chen N, Zhou M, Dong X, Qu J, Gong F, Han Y, et al. Epidemiological and clinical characteristics of 99 cases of 2019 novel coronavirus pneumonia in Wuhan, China: a descriptive study. Lancet. 2020 Feb 15;395(10223):507513.Pubmed PMID: 32007143.

[47]. Tang YW, Schmitz JE, Persing DH, Stratton CW. Laboratory diagnosis of COVID-19: current issues and challenges. J Clin Microbiol. 2020 May 26;58(6):e00512-20

[48]. Yang W, Yan F. Patients with RT-PCR-confirmed COVID-19 and normal chest CT. Radiology. 2020 May;295(2):E3.

[49]. Udugama B, Kadhiresan P, Kozlowski HN, Malekjahani A, Osborne M, Li VY, et al. Diagnosing COVID-19: the disease and tools for detection. ACS nano. 2020 Mar 30;14(4):3822-35.

[50]. Tahamtan A, Ardebili A. Real-time RT-PCR in COVID-19 detection: issues affecting the results. Expert Rev. Mol. Diagn. 2020;20(5):453-454.

[51]. Chan JF, Yip CC, To KK, Tang TH, Wong SC, Leung KH, et al. Improved Molecular Diagnosis of COVID-19 by the Novel, Highly Sensitive and Specific COVID-19-RdRp/Hel Real-Time Reverse Transcription-PCR Assay Validated In Vitro and with Clinical Specimens. J Clin Microbiol. 2020 Apr 23;58(5):e00310-20.Pubmed PMID: 32132196.

[52]. Xu J, Li Y, Gan F, Du Y, Yao Y. Salivary Glands: Potential Reservoirs for COVID-19 Asymptomatic Infection. J Dent Res. 2020 Jul;99(8):989.Pubmed PMID: 32271653.

[53]. Ather A, Patel B, Ruparel NB, Diogenes A, Hargreaves KM. Coronavirus disease 19 (COVID-19): implications for clinical dental care. J. Endod. 2020 Apr 6.

[54]. CDC. Coronavirus Disease 2019 (COVID-19). Centers for Disease Control and Prevention. Published February 11, 2020. Accessed April 21, 2020.

[55]. Personal protective equipment and Covid-19 - COVID-19 Research Collaborations. Accessed September 11, 2020.

[56]. Turer RW, Jones I, Rosenbloom ST, Slovis C, Ward MJ. Electronic personal protective equipment: A strategy to protect emergency department providers in the age of COVID-19. J Am Med Inform Assoc. 2020 Jun 1;27(6):967971.Pubmed PMID: 32240303.

[57]. Peng X, Xu X, Li Y, Cheng L, Zhou X, Ren B. Transmission routes of 2019$\mathrm{nCoV}$ and controls in dental practice. Int J Oral Sci. 2020 Mar 3;12(1):9.

[58]. Seto WH, Tsang D, Yung RW, Ching TY, Ng TK, Ho M, et al. Advisors of Expert SARS group of Hospital Authority. Effectiveness of precautions against droplets and contact in prevention of nosocomial transmission of severe acute respiratory syndrome (SARS). Lancet. 2003 May 3;361(9368):1519-20.Pubmed PMID: 12737864 